\title{
Helically corrugated waveguide microwave pulse compression experiments
}

(Poster Presentation)

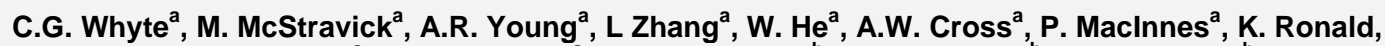 \\ C.W. Robertson ${ }^{a}$, A.D.R. Phelps ${ }^{a}$, S.V. Samsonov ${ }^{b}$, S.V. Mishakin ${ }^{b}$, G.G. Denisov ${ }^{b}$, \\ V.L. Bratman ${ }^{b}$ and N.G. Kolganov',

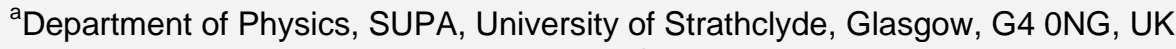 \\ bInstitute of Annlied Phvsics Ruscian Academv of Sciences Nizhnv Novonorod 603950 Ruscia
}

\section{Summary Paper}

A high microwave pulse compressor based on a large diameter 5-fold helical waveguide structure was studied. The eigenwave dispersion was calculated using numerical and analytical techniques and compared with experimental measurements. The results of 5-fold helically corrugated waveguide microwave pulse compression experiments will be presented.

\section{Microwave pulse compression experiment using a 3-fold helically corrugated waveguide}

$\mathrm{X}$-band microwave pulse compression has applications in radar technology and plasma diagnostics [1]. The 3-fold helical corrugation [2] couples a pair of partial modes of the smoothbore circular waveguide having significantly different group velocities: $\mathrm{TE}_{2,1}$ mode close to cut-off and $\mathrm{TE}_{1,1}$ far from cut-off. At non-zero amplitude of corrugation, resonant coupling between the modes occurs when their axial and azimuthal wave numbers satisfy the Bragg conditions resulting in the operating eigenmode [3]. As a result, in the frequency region of their resonant coupling, the resultant eigenmode has a strong frequency dependent group velocity which is favourable for pulse compression [4]. A modulated, frequency-swept microwave pulse was created using an arbitrary waveform generator and vector signal generator.
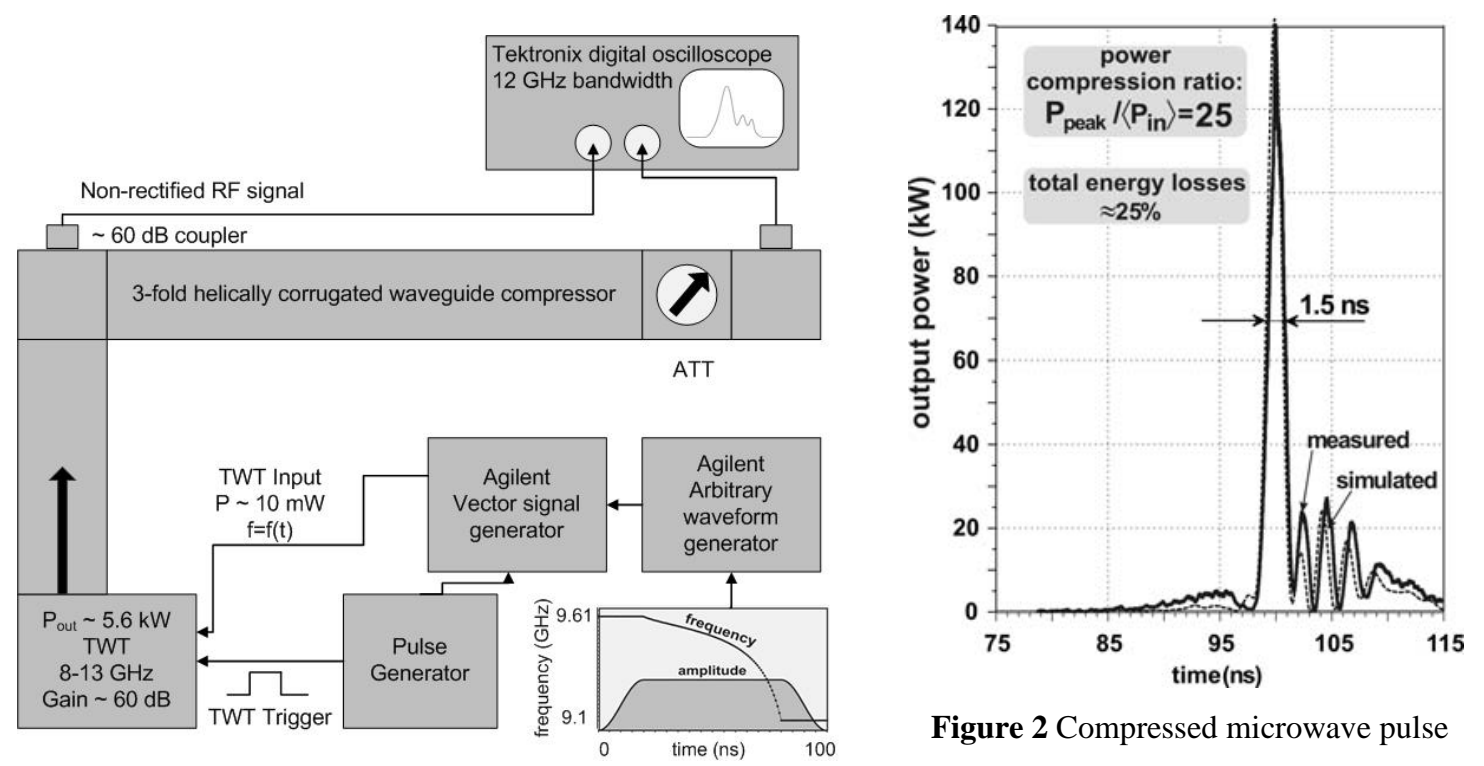

Figure 1Experimental set-up

Figure 2 Compressed microwave pulse

The pulse was then amplified using a high-power 7kW TWT (TMD PTC6321), with the pulse amplitude and pulse duration measured prior to entry of the compressor. The frequency sweep was optimised to be $9.61-9.10 \mathrm{GHz}$, while the input pulse was found to have an average power of 5.5kW and duration of 80ns. The pulse was compressed in the 3-fold helically corrugated waveguide compressor of $2.08 \mathrm{~m}$ in length. The output power of the pulse from the helix was attenuated to match that of the input pulse, allowing the compression ratio to be determined via the ratio of the attenuation used for the compressed and uncompressed pulses. The compressed pulse was found to have a duration of 1.5 ns, with a peak power of $135 \mathrm{~kW}$, giving a power compression ratio of 25. The compression efficiency was calculated to be $75 \%$. 


\section{5-fold helically corrugated waveguide}

To enhance the power capabilities a more overmoded larger diameter 5-fold copper helical waveguide structure system was designed to compress microwave pulses with frequency modulation within the interval of 9.61-9.10GHz and simultaneously provide low reflection of input radiation within a frequency interval of 8-10 GHz. It is capable of handling GW levels of power. The set-up (Figure 3) consists of an input cone to introduce the radiation from the input source, a mode converter to convert the input mode into the operating mode of the compressor, an intermediate cone to transport the radiation keeping acceptable mode purity and a pulse compressor to compress the frequency modulated radiation. At the input cone the radiation will be in the form of a left-handed circularly polarized $\mathrm{TE}_{1,1}$ mode. The output radiation from the mode converter will be in the form of a right-handed circularly polarized $\mathrm{TE}_{3,1}$ mode which enters the compressor to couple with the close-to-cutoff $\mathrm{TE}_{2,2}$ mode. The reciprocal set-up is required on the output side of the pulse compressor to obtain the compressed $\mathrm{TE}_{1,1}$ wave.

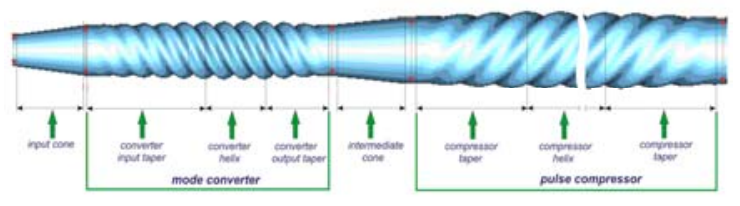

Figure 3 5-fold microwave pulse compression

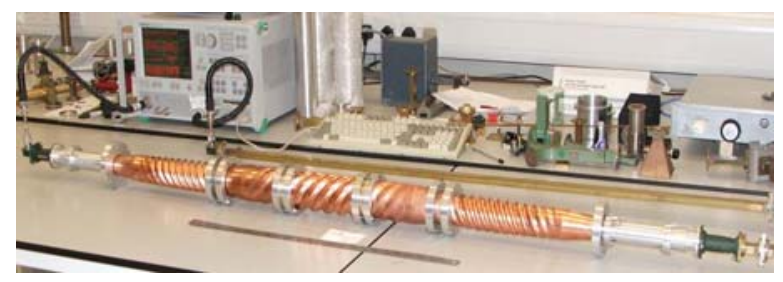

Figure 4 Vector network analyzer set-up

The dispersion characteristics of the eigenwave were studied using 3 different techniques; numerical (CST microwave studio-MWS), analytical (coupled wave theory), and experimental (vector network analyzer). Firstly, a single period of the 5-fold helix was constructed in MWS. Using the eigenmode solver within MWS the eigenmode was simulated (Figure 5 ) for various boundary phase angles $(\phi)$ ). The corresponding axial wavenumber $k_{z}$ was calculated from, $k_{z}=\phi / d$. Using coupled wave theory [3] the eigenwave dispersion was calculated for the interaction of the two electromagnetic waves present in the 5-fold helix; the $\mathrm{TE}_{3,1}$ and $\mathrm{TE}_{2,2}$. A vector network analyzer (Figure 4) was used to measure the phase of the eigenwave. The VNA was calibrated using a line-reflect-line method. The S21 parameter was recorded as a function of frequency. A measurement was taken when the section of 5-fold helix was present and similar measurement was taken without the helix present. The difference in the two measurements was analyzed digitally. The results from the three techniques shown in Figure 6 are in good agreement with one another.

a)

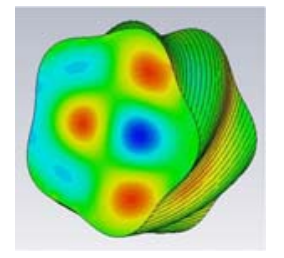

b)

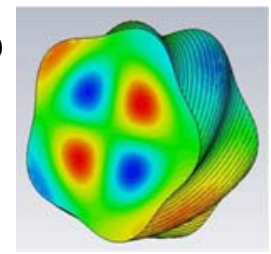

Figure 5 Eigenmode profile of (a) transverse electric field (b) axial magnetic field

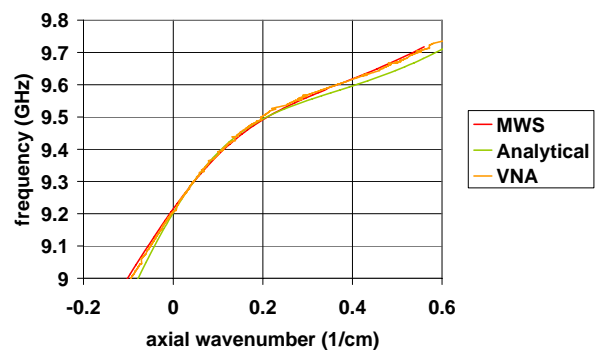

Figure 6 Figure 7 Eigenwave dispersion results

The constructing of the full length (approx. $3 \mathrm{~m}$ ) of the 5-fold pulse compressor has been completed. Sweep-frequency based microwave pulse compression experiments at $\mathrm{mW}$ and $\mathrm{kW}$ levels of power will be presented.

\section{References}

[1] S.V. Samsonov, A.D.R. Phelps, V.L. Bratman, et al., Phys. Rev. Lett., Vol 92, 118301, (2004)

[2] G. Burt, S.V. Samsonov, K. Ronald, et al., Phys. Rev. E, Vol 70, 046402, (2004)

[3] G. Burt, S.V. Samsonov, A.D.R. Phelps, et al., IEEE Trans. Plasma Sci., Vol 33, no. 2, pp. 661-667, (2005)

[4] M. McStravick, S. Samsonov, K. Ronald, S. Mishakin et al, Journal of Applied Physics, Vol 106, 0544908, (2010) 
2nd Annual Seminar on Passive RF and Microwave Components 